\title{
Study on application of SDN technology in electric power communication network
}

\author{
Hongfei $\mathrm{Xu}$, Lingfeng Wen, Xifan Nie, Ningzhe Xing and Yajie $\mathrm{Pu}^{\mathrm{a},{ }^{*}}$ \\ State Grid Jibei Information \& Telecommunication Company, Beijing, China \\ a, ${ }^{*}$ wshbupt@163.com
}

Keywords: SDN, electric power, data center, architecture.

\begin{abstract}
With the development of electric power communication network, SDN is gradually introduced in the next generation electric power communication network. The application of SDN in power data center is analyzed, and architecture of power data center based on SDN is provided, finally two ways of evolving from traditional power data center to SDN based power data center are provided.
\end{abstract}

\section{Introduction}

SDN (Software Defined Network) is a network technology architecture based on software programmable technology [1]. Its goal is to use software technology to optimize the network technology and deploy scheme, to improve the intelligence and automation ability of the network, to realize the agility of the network, the ability to optimize the resources [2-5]. SDN network through a software-based control plane to achieve standardized, distributed forwarding plane management and control; control plane and forwarding plane are separated; provide open north and south interface; provide flexible programmable capacity for the network. In the next generation of power communication network, the introduction of SDN network can improve network architecture, business support capabilities, operation and maintenance efficiency of the traditional power communication network

\section{Application of SDN}

In the power industry, the introduction of SDN technology in data center is to achieve intelligent and automated network, to realize software-centric, customer-oriented, business and service network, the basic functional requirements include service automation, networking Expansion, resource scheduling, operation and maintenance automation.

\subsection{Service Automation}

Through connect with the power cloud management platform, the SDN-based power data center can automatic sense the network resource demand in the power cloud service, and can optimize the power service network design, automatically deploy the solution, and feedback to power cloud management platform. When business requirements change, the SDN network can dynamically adjust the network design to provide capabilities on-demand. After the termination of the service, the SDN network can timely recover the network resources allocated for the service, and feed back to the power cloud platform, to provide historical data.

Main functions include:

- Ability to dynamically sense the network requirements of the power cloud service

- Ability to optimize the design of the electricity cloud service network

- Ability to automatically instantiation of the power cloud service network

- Ability to presentation of the electricity cloud service network in real time

- Ability to adjustion on-demand of the power cloud service network

- Ability to recover of network resources in time

- Ability to reliably record log of the power cloud service network 


\subsection{Scalability}

From a business point of view, SDN-based power data center networks can support an increasing number of users. From the device point of view, SDN-based power data center network should be able to support multi-vendor SDN equipment interoperability, and can be compatible with existing power data center equipment. From the system construction point of view, the control plane should have good scalability. From the network planning point of view, based on the SDN power data center network should be able to support cross-regional interconnection between data centers. Through the above capabilities, SDN-based power data center can provide a complete solution.

The main functions include:

- Support massive virtual machine access

- Compatible with the existing forwarding equipment in power data center network

- Support interworking of multi-vendor SDN equipment

- Support hybrid deployment of software, hardware equipment

- Guarantee the scalability of control plane

- Support interoperability of inter-regional power data center

\subsection{Resource Scheduling}

SDN-based power data center network can realize real-time scheduling of the underlying resources, to meet the business reqirements of the flexible network. The SDN control plane can maintain the global resource view of the power data center in real time, and adjust the forwarding logic of the device in real time based on real-time link bandwidth and service requirements. SDN data plane forwarding equipment can adjust in real time according to the control plane strategy, the forwarding table, queue and other resources. Taking into account the integration with NFV, SDNbased power data center network can support customized service to guide traffic through the appropriate service node.

The main functions include:

- Ability to maintain global resource views in real time

- Monitor link bandwidth in real time

- Dynamically adjust traffic forwarding path

- Support different queue priorities

- Guided service traffic of the service node by the location

\subsection{Operation and Maintenance Automation}

SDN-based power data center network have a good automated operation and maintenance capabilities, reduce the network administrator's manual configuration work as much as possible , automatically fault location and fault recovery when a network failure occurs.

The main functions include:

- Support the plug and play of the forwarding device

- Ability to provide deployment script for control plane devices

- Ability to visualize physical traffic and service traffic

- Comprehensive statistics and analysis of flow

- Ability to automatically deploy flexible ACL policies

- Automatically detect most of the power network fault

- Automatically complete most of the power network failure recovery

\subsection{Architecture of Power Data Center}

SDN-based power data center network architecture is divided into application layer, layout layer, control layer and forwarding layer, including the corresponding function of the network management to achieve: 


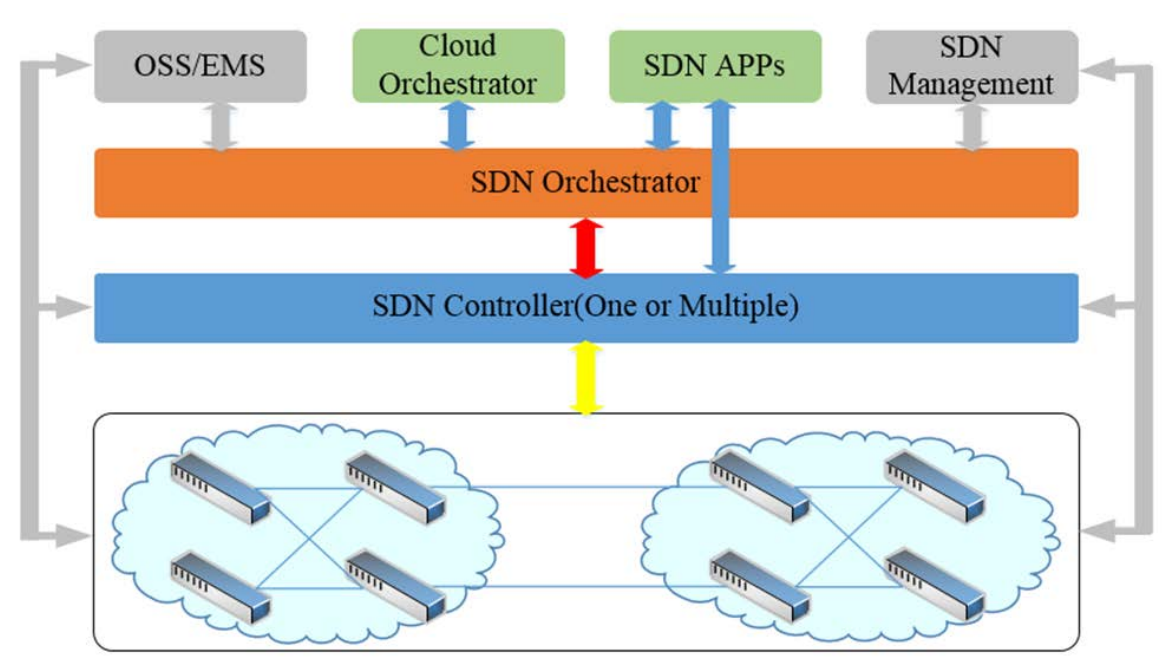

Figure 1. Architecture of power data center based on SDN

Application layer: including the power cloud management platform and SDN applications, which include traditional network management and SDN network management part. The power cloud management platform communicates with the layout layer through the network resource interface, and conveys the demand description of the cloud service to the network. The SDN application communicates with the control layer and passes the application logic. The communication between Cloud management platform, SDN application, the layout layer and the control layer follow the standard interface protocol, such as Restful API, Java, Python and so on.

Layout layer: mainly includes SDN arranger, responsible for the coordination of multiple SDN control domain, to achieve automatically open and deploy of the power cloud business network resources. By interacting with the upper-level cloud management platform or the SDN application, the SDN arranger automatically senses the service requirements of the network resources, and combines the logical resource views of the control domains to design the service plan and uses the service primitives Way distribution to the following controller. The communication between the SDN scheduler and the power cloud management platform, SDN application and controller adopts the standard interface format or protocol. The communication between the SDN arranger and the power cloud management platform can adopt the Restful API and so on. The communication between the SDN arranger and the controller can adopt Netconf Wait.

Control layer: mainly includes one or more SDN controllers to meet the demand of network resources of power cloud service and SDN application, which are responsible for scheduling the network resources in each control domain. On the one hand, the SDN controller interacts with the SDN scheduler or SDN application through the northbound interface, reports the logical network resource view, and receives the service primitives and application logic. On the other hand, the controller obtains the network resource view in the domain through the south interface protocol, and realizes the scheduling of the network resources according to the service primitives or application logic. The northbound interface can be Restful API, Netconf, Java, Python, etc., to the south interface protocol can be OpenFlow, PCEP, SNMP and so on. The communication between the SDN controller and the network management system adopts the standard interface format or protocol of the traditional network management or SDN network management.

Forwarding layer: mainly includes SDN forwarding device, which may include some traditional network forwarding devices. The SDN forwarding device receives the forwarding logic from the SDN controller through the southbound interface protocol, and forwards the traffic according to the corresponding forwarding logic. The southbound interface protocol can adopt OpenFlow, PCEP, and SNMP. At the same time, SDN forwarding layer device can also have some autonomy control logic, so that the local part of the forwarding decision. The communication between the forwarding layer device and the network management system adopts the standard interface format or protocol of the traditional network management or SDN network management. 
Network management function: the SDN equipment and the traditional network equipment in the data center will coexistence in the long-term, SDN-based power data center network management include traditional network management and SDN network management. The SDN network management system can be built independently or integrated with the traditional network management system. The SDN network management may have interfaces with the application layer, the scheduling layer, the control layer and the forwarding layer. The SDN network management layer may communicate with the application layer or the forwarding layer. The specification of the SDN network management Interface design is not explicitly required. In addition, part of SDN network management may exist in the application layer (such as SLA security, etc.), the layout layer (such as service scheduling, service adaptation, etc.) and control layer (such as path switching) The functionality of the network management and other parts of the architecture is not explicitly defined.

\subsection{Evolution to SDN Power Data Center}

Traditional power data center networks have two ways to evolved into SDN power data center networks: from traditional power data centers to SDN power data centers, and from traditional power data centers to Overlay SDN power data centers.

\subsubsection{Evolution to Pure SDN Power Data Center}

Traditional power data center network migrate to pure SDN power data center network have two ways: direct upgrade and phased migration. Through the addition of SDN control plane agents (such as OpenFlow, BGP) on the existing network devices, the existing control mechanism on each device is eliminated. SDN controller realizes the direct evolution way of the traditional network to SDN network.

Phased migration mode is as follows, in the first stage only the edge of the data center network equipment upgrade to SDN, the SDN controller control transponder ID mapping and mapping in the overlay network, the core network equipment still forward based on the traditional forwarding table, combined with traditional equipment deployed in the current network in the phased deployment of SDN equipment. The original device and the SDN controller responsible for network operation and maintenance together. In the second stage, upgrade the network core equipment gradually, eliminate the original control mechanism once the traditional network complete the migration to the SDN network equipment.

SDN-based forwarding equipment divided into three categories: legacy equipment, pure SDN equipment and Hybrid equipment. Legacy devices refer to the forwarding devices in traditional networks. The control logic of the legacy devices is integrated locally. The control logic of pure SDN devices is implemented by the remote controller. Hybrid devices are devices that combine traditional control logic and SDN control plane agents.

The evolution way applied for internal and external of the SDN power data network evolution.

\subsubsection{Evolution to Overlay SDN Power Data Center}

In overlay SDN network, the forwarding logic is put on the edge of the network server and power data center exit gateway, or other equipment such as TOR, switches and other equipment, does not involve in traditional power data center network transformation, can easily achieved power SDN upgrade between network and power data centers in the data center. Traditional power data center networks built using Overlay technology are proposed to migrate using the Phased Migration method.

SDN evolution in the power data center is shown in Figure2. In a traditional power data center network, each virtual machine needs to point the gateway to a Layer 3 convergence switch or a Layer 3 router. The use of Overlay SDN solution does not need to transform any network equipment in the data center, VRS is installed in each physical server Hypervisor, put the complex forwarding logic on the server, and increase the strategy engine and controller and other control unit in the network, can achieve transformation within the power data center. 

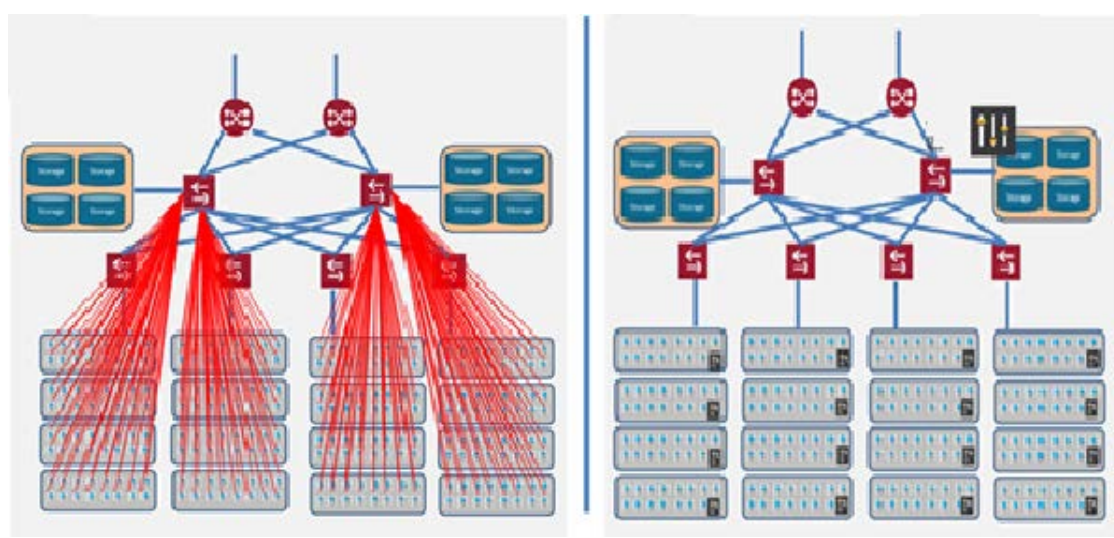

Figure 2. Evolution of SDN in power data center

The SDN evolution pattern between power data centers is shown in Figure 2. In the transformation of the network between the power data centers, only the gateway needs transformation, which can support VLAN, VxLAN, NvGRE, and support existing VPLS, EVPN, VxLAN, etc. the interoperability between the data centers is achieved without any transformation.

The requirements of servers in SDN evolution is shown in Figure 2. VRS software installed for different hypervisors (such as Vmvare, KVM, XEN), the server can have forwarding logic, including IP forwarding, speed limiting, ACL / FW, VxLAN package and so on. For a server that can not install the Hypervisor, VRS functionality need to be implemented in corresponding TOR switcher, to complete the Overlay SDN network access.

\section{Conclusions}

The application of SDN in power data center is analyzed, and architecture of power data center based on SDN is provided, finally two ways of evolving from traditional power data center to SDN based power data center are provided.

\section{References}

[1] YANG Xu, ZHOU Ye, LI Yong. Software-defined data center networks [J]. ZET Technology Journal, 2014, 20(5): 50-54.

[2] Tran Manh Nam; Nguyen Huu Thanh, Energy-aware routing based on power profile of devices in data center networks using SDN, Electrical Engineering/Electronics, Computer, Telecommunications and Information Technology (ECTI-CON), 2015 12th International Conference on, 2015, Pages: $1-6$.

[3] QIAO Lin, YIN Xiao-hua, DI Zhuo, et al. Research on SDN network architecture for electric power big data platform [J]. Electric Power Information and Communication Technology, 2015, 13(1): 21-26.

[4] WANG Yi, ZHANG Bao-ying, WANG Hai. Application research on software defined network in power grid enterprises [J]. Electric Power Information and Communication Technology, 2015, 13(4):73-77.

[5] Yiming Yu; Yongli Zhao; Jie Zhang, Using SDN in centralized data centers in power communicationnetworks for disaster recovery,2014 12th International Conference on Optical Internet 2014 (COIN), 2014, Pages: 1-2 